\title{
Experimental and Measurement Methods for the Small-Scale Model Testing of Lateral and Torsional Stability
}

\author{
Jong-Han Lee ${ }^{1)}$, Yong Myung Park ${ }^{2), *}$, Chi-Young Jung ${ }^{3)}$, and Jae-Bong Kim ${ }^{3)}$
}

(Received September 15, 2016, Accepted March 23, 2017, Published online May 19, 2017)

\begin{abstract}
Tests of the lateral and torsional stability are quite sensitive to the experimental conditions, such as support conditions and loading system. Controlling all of these conditions in a full-size test is a very challenging task. Therefore, in this paper, an experimental measurement method that can control the experimental conditions using a small-scale model was proposed to evaluate the lateral and torsional stability of beams. For this, a loading system was provided to maintain the vertical direction of the load applied to the beam, and a support frame was produced to satisfy the in-plane and out-of-plane support conditions. The experimental method using a small-scale model was applied successively to the lateral and torsional behavior and stability of I-shaped beams. The proposed experimental methods, which effectively accommodate the changes in the geometry and length of the beam, could contribute to further experimental studies regarding the lateral and torsional stability of flexural members.
\end{abstract}

Keywords: lateral torsional stability, critical load, in-plane behavior, out-of-plane behavior, lateral deformation, rotational angle.

\section{Introduction}

The lateral and torsional stability is the main issue for the safety of structures and users from the stages of design to construction in the field of architectural and civil engineering (Kalkan 2014; Hurff and Kahn 2012; Lee 2012a, b; Lee et al. 2016; Kim et al. 2016; Hou and Song 2016; Petrone et al. 2016; Ramin and Fereidoonfar 2015; Srikar et al. 2016). Analytical investigations into the lateral and torsional stability have been performed to develop a classical stability theory based on the steel structural members (Horne 1954; Salvadori 1955; Timoshenko 1956; Timoshenko and Gere 1961). Several studies (i.e., Talbot and Dhatt 1987; Rengarajan et al. 1995; Li et al. 2002; Darilmaz 2011; Brsoum and Gallagher 1970; Fafard et al. 1987) developed finite element analysis using shell and plate elements for buckling analysis, which requires large geometric nonlinearity and bifurcation.

For slender and long precast concrete flexural members, most studies (Hansell and Winter 1959; Sant and Bletzacker 1961; Massey 1967; Siev 1960; Revathi and Menon $2007 \mathrm{a}, \mathrm{b}$ ) focused on the classical formulation to propose the

\footnotetext{
${ }^{1)}$ Department of Civil Engineering, Daegu University, Gyeongsan 38453, Republic of Korea.

${ }^{2)}$ Department of Civil Engineering, Pusan National University, Busan 46241, Republic of Korea.

*Corresponding Author; E-mail: ympk@pusan.ac.kr

${ }^{3)}$ Seismic Simulation Test Center, Pusan National University, Busan 46241, Republic of Korea.
}

Copyright $\odot$ The Author(s) 2017. This article is an open access publication weak-axis flexural rigidity and torsional rigidity associated with the lateral and torsional instability of concrete beams. The lateral and torsional stability of the slender and long concrete beams are also strongly affected by initial geometric imperfections. Recently, Kalkan (2014) and Hurff and Kahn (2012) have attempted to evaluate the influence of the initial lateral imperfection in the lateral and torsional buckling of slender, rectangular reinforced and prestressed concrete beams. In addition, Lee (2012a, b) examined the initial lateral deformation caused by environmental thermal effects to evaluate the lateral behavior and stability of bridge I-girders using three-dimensional finite element analysis and experimental data. Moreover, such these imperfections along the length of the beam are considered to be among the main causes of the rollover instability collapse of bridge girders (Oesterle et al. 2007; Zureick et al. 2005), which is the main issue during the construction of concrete and precast beams.

On the other hand, the experiment of lateral and torsional stability requires special caution to minimize geometric imperfections and material irregularities in the manufacturing and testing processes of a specimen, as well as to implementing support and loading conditions. That is, the loading system applied to the beam should retain its vertical direction throughout the testing while allowing longitudinal (in-plane) and transverse (out-of-plane) transitional and flexural movements of the beam. In addition, a lateral support condition should be provided to restrain the transitional and rotational movements but allow flexural behavior in both the longitudinal and lateral directions. Such support and loading conditions are extremely difficult to perform completely, particularly in a full-size beam test. A slight deviation in the support and loading conditions completely changes the experimental results. Moreover, the lateral and 
torsional buckling of a beam induces lateral and vertical deformation combined with a twisting rotation of the beam.

Therefore, this study presented a small-scale experimental method to implement the support and loading conditions and minimize the experimental errors when assessing the lateral and torsional stability of a beam. An experimental frame was provided to install a specimen and satisfy the loading and support conditions. The frame was also designed to adapt the changes in the loading and support conditions, as well as the size and length of the beam. A loading transfer system was then provided to retain the initially vertical orientation of the load to a beam undergoing the coupled deformation and rotation. The support condition was also designed to satisfy the in-plane and out-of-plane restraint and movement conditions. Finally, the instrumentation was carried out to measure the variations in the deformation and angle and longitudinal strain in the beam. The lateral and vertical deformations and a rotational angle of the beam were determined from the coupled transformations using the measurement method, which was proposed initially by Zhao et al. $(1994,1995)$ and later modified by Stoddard (1997), and applied successively by Kalkan (2009) and Hurff (2010). In the present study, the measurement method was expanded to allow easier and more useful instrumentation and data processing. As a result, the proposed experimental and measurement methods using a small-scale model can be applied easily and effectively for the lateral and torsional stability testing of flexural members to evaluate the critical load and the influencing factors, such as geometric, support, and loading conditions, in the field of engineering.

\section{Objectives and Significance}

Tests of the lateral and torsional stability are quite sensitive to the experimental conditions, such as support condition, loading system, and material nonlinearity. Moreover, the lateral and torsional buckling test using a full-size beam requires considerable time and effort to control all these conditions. Even with careful consideration of these factors, unexpected errors, such as a deviation from the designed support, loading, and material conditions, could completely change the experimental results. Therefore, the appropriate loading, support, and material conditions in a full-size test are extremely difficult to achieve. Therefore, this study presented a small-scale experimental method that can control and maintain the loading and support conditions in the lateral and torsional stability tests of beams. The support system was developed to satisfy both the in-plane and out-of-plane restraints and movement conditions. The loading system was designed to retain its initial vertical direction throughout the course of loading and allow the lateral and torsional movement of the specimen at the application point of the load. The experimental method was applied successively to the lateral and torsional buckling of small-scaled I-shaped beams. The proposed experimental methods, which effectively accommodate the changes in the geometry and length of the beam, could contribute to further experimental studies regarding the lateral and torsional stability of flexural members.

\section{Experimental Program}

\subsection{Beam Specimen}

The material selected for the small-scale beam model was polycarbonate, which is strong and durable, as well as linearly elastic. Polycarbonate allows easy specimen preparation and the installation of various sensors, such as strain gauges, displacement meters, and accelerometers, for an experiment. In addition, with the advantage of the linear elasticity of a material, various experiments and explicit interpretation are available to evaluate the influential factors on the lateral and torsional stability. The modulus of elasticity, which plays a decisive role in determining the buckling load, was analyzed using the relationship between the applied load and vertical deflection measured at the middle of the test beam.

The size of the model beam was scaled down using the aspect ratios of the section and length of a typical precast beam, AASHTO Type VI beam.

Figure 1 provides the detailed dimensions of the crosssection of the AASHTO Type VI and scaled model beam. The cross-section of the testing specimen neglected the fillets at the connection of the web and flanges for easier manufacturing, as shown in Fig. 1b. The sectional properties of the model beam are determined to be similar to those of the AASHTO Type VI to minimize the influence of the fillets in the lateral and torsional behavior of the beam. The ratio of the height-to-width of the cross-section of the Type VI and model beam was 1.71 and 1.80 , respectively. The thickness-to-width ratio of the cross-section in the top flange of the Type VI and model beam was 0.17 and 0.15 , respectively. The size of the bottom flange of the model beam was designed to be the same as that of the top flange of the beam. Therefore, the test beam is doubly symmetrical; thus, the center of geometry and shear force coincides. The ratio of the second moment of the section in the weak axis to that in the strong axis of the Type VI and model beam was calculated to be 0.09 and 0.10 , respectively.

\subsection{Support Conditions and Frame}

Lateral and torsional stability testing needs to satisfy both the in-plane (strong-axis) and out-of-plane (weak-axis) support conditions. Figure 2 shows the support boundary conditions and a support frame designed for the lateral and torsional stability testing of a beam. The simple support condition was implemented using a roller that restrains the vertical and lateral translations but allows in-plane flexural rotation of the beam. Furthermore, the lateral and torsional restraints should be provided along the depth of the beam at the location of the simply-supported condition. On the other hand, the lateral support, which restrains the lateral translation and twisting rotation, can permit rotations about the strong and weak axes.

Therefore, lateral support and support frames were designed using a ball caster and circular rod, shown in Fig. 2. The circular support rod can move laterally to adjust the width of the beam and is fastened to act as a lateral 
(a)

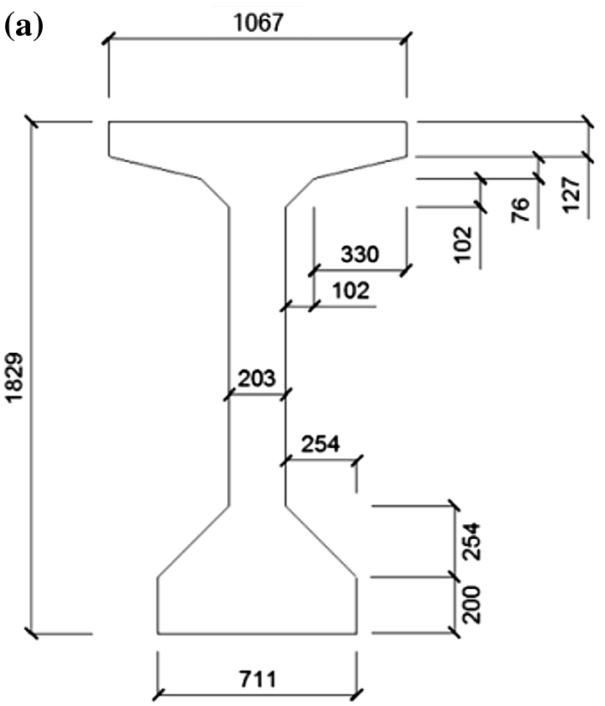

(b)

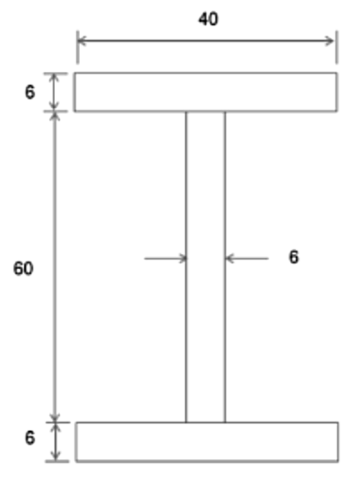

Fig. 1 Cross-section of the a AASHTO Type IV and $\mathbf{b}$ test beam (units: $\mathrm{mm}$, not to scale).

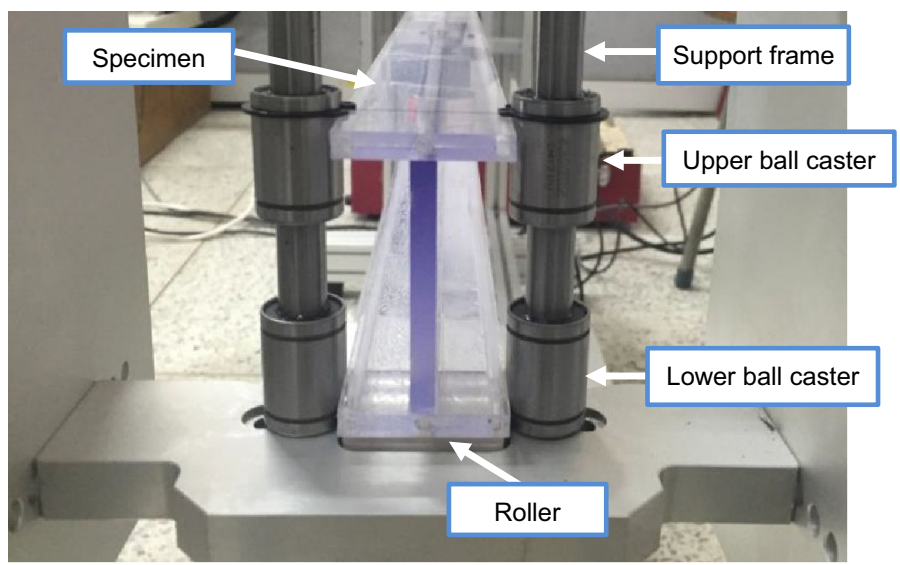

Fig. 2 Strong-axis (in-plane) and weak-axis (out-of-plan) supports.

support condition. The ball caster, which is in contact with the side surface of the top and bottom flanges of the beam, contains grease to minimize friction resistance at the contact surface of the ball caster and the circular rod. The top and bottom ball casters, which provide lateral support to the top and bottom flanges of the beam, respectively, is free to move up and down, as well as rotate when the rotations about the strong and weak axes occur in the beam. Therefore, the inplane and out-of-plane rotations of a beam are unconstrained at the support locations.

\subsection{Vertical Loading System}

The lateral and torsional stability is strongly dependent on the vertical loading system along with the support condition. The vertical load applied to a beam should retain its vertical direction throughout the course of loading even when lateral and torsional behavior is observed. In addition, the loading transfer mechanism should not have any resistance on the lateral deformation and torsional rotation at the application point of the load. Previous studies using lateral and torsional buckling experiments focused on full-scale size beams. The loading mechanisms used in previous studies were either distributed or point loads. König and Pauli (1990) utilized steel and water weights as the distributed load mechanism to perform the lateral and torsional buckling experiment in reinforced and prestressed concrete beams. The distributed load mechanism has limitations on space, logistics, and safety, which has rarely been applied in the test (Kalkan and Hurff 2012). Therefore, most of the lateral and torsional buckling experiments have attempted to use a point load mechanism to retain the initial vertical and concentrical position of the applied load without its resistance to the lateral and torsional restraints of the test beam at the loading point. Jensen (1978) initially applied a point load for the lateral and torsional buckling experiment but had difficulties in retaining the position of the applied load. Therefore, many studies (i.e., Hansell and Winter 1959; Sant and Bletzacker 1961; Massey 1967; Siev 1960; Revathi and Menon $2007 \mathrm{a}$, b) used roller bearing and ball-and-socket joint mechanisms to minimize the resistance to the lateral and rotational deformations of the test beam at the loading point during the test. Kalkan and Hurff (2012) indicated that the roller bearing mechanism could induce load eccentricity due to some difference between the centroid of the applied load and the beam when the specimen undergoes lateral deformation. Therefore, another load mechanism using a gravity 
(a)

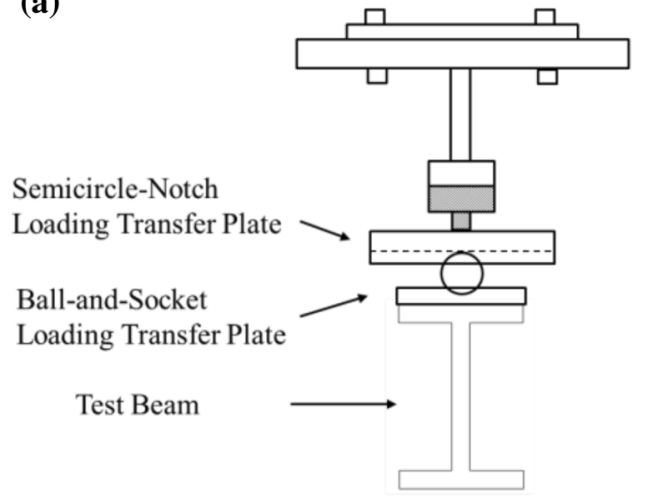

(b)

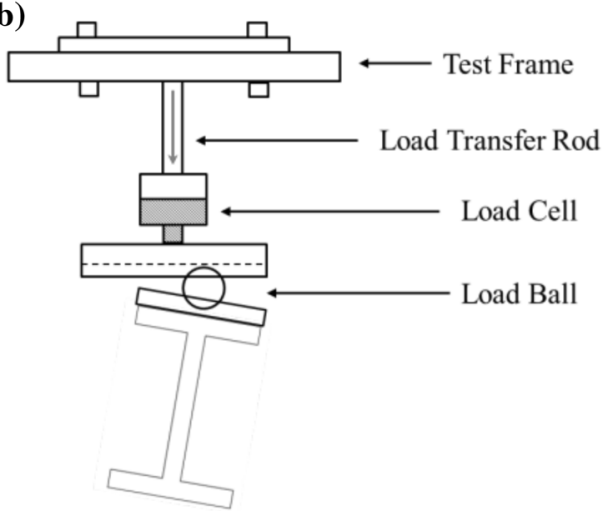

Fig. 3 Design of the loading system for the a undeflected and $\mathbf{b}$ deflected configurations of a beam.

load simulator, which was originally designed by Yarimci et al. (1967) to apply a vertical load to a full-scale frame experiment, was used in the lateral and torsional buckling experiments on full-scale steel I-beams (Yura and Phillips 1992; Helwig et al. 2005), composite I-beams (Stoddard 1997), and reinforced concrete beams (Kalkan 2014; Hurff and Kahn 2012). Nevertheless, the gravity load simulator also inclined the loading unit during the test, which requires an additional control mechanism to maintain the vertical line of the load.

Based on previous studies, this study devised and proposed a vertical loading system based on the roller mechanism, as shown in Fig. 3. The load applied from the loading unit, mounted to the top of the test frame, is transferred to the beam through the load transfer rod and plates. The top load transfer plate, which is connected directly to a load cell device, was designed to allow free lateral deformation of the beam using a semicircle notch along the width of the beam without resistance. To retain the vertical and concentric direction of the load to the beam at the loading point, the load was transferred to the beam with the help of a ball-and-socket joint. The steel ball can be free to rotate in the ball-and-socket joint and semicircle notch, and also move laterally when lateral and torsional movements of the beam begin. The size of the steel ball was determined to have some vertical distance between the top and bottom transfer plates; thus the beam was free to rotate without any intervention induced by the plates. In addition, the interface between the steel ball and the top and bottom plates was greased to minimize friction at the loading point. Figure 4 shows a picture of the loading transfer system and the small-scaled model beam. In addition, the loading system was designed easily to move along the top of the test frame using screw bolts, and it can be secured firmly to a target location. Therefore, the proposed loading system can be easily applicable to the third-point loading as well as the fourth-point loading, where two vertical loads are applied simultaneously to the beam.

\section{Experimental Measurement}

\subsection{Instrumentation and Measurements}

The small-scaled I-beam, $950 \mathrm{~mm}$ long between the supports with the cross-section, shown in Fig. 1b, was

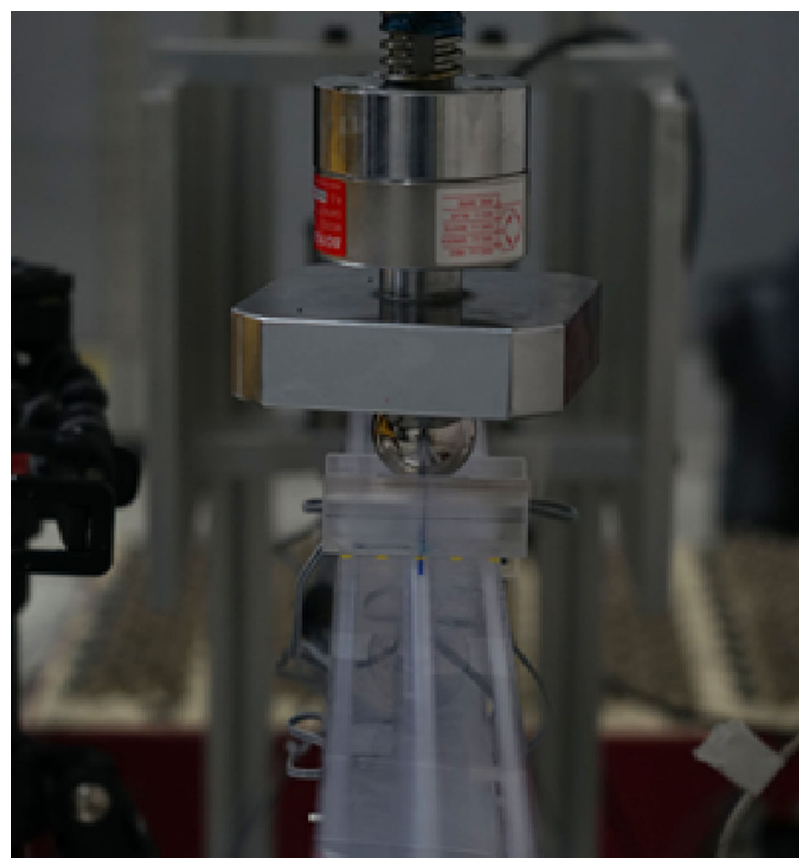

Fig. 4 Loading transfer system.

installed on the in-plane and out-of-plane support conditions proposed in this study. As described previously, the beam specimen installed on the support boundary condition is shown in Fig. 2. A vertical load was then applied to the middle of the top surface of the beam, and the applied load was measured using a load cell device installed in the vertical loading system, shown in Fig. 4. The deflections and rotation of the beam were measured using wire potentiometers installed at the mid-span of the beam. The initial vertical line of the potentiometers begins to incline as the lateral and torsional deformations occur. That is, because lateral and vertical deflections are combined with a twisting rotation of the beam, the deflections and rotation cannot be obtained directly from the measurements from the potentiometers. Therefore, in this study, the measurement technique, which was developed by Zhao et al. (1994, 1995) and modified later by Stoddard (1997), was employed to acquire the independent lateral and vertical deflections and twisting rotation. The technique requires a total of three wire potentiometers: two 
potentiometers installed on the top and bottom of a specimen in the lateral direction, and one potentiometer installed vertically at the bottom of the specimen. Figure 5 shows a picture of the two wire potentiometers connected to the top and bottom of the test beam. In addition to the lateral wire potentiometers, two vertical potentiometers are installed in the right and left sides of the bottom flange of the beam. In fact, one vertical potentiometer is sufficient to convert the couple deflections and rotation from the measurement. In this study, another potentiometer was used in the bottom of the beam to assess the distortion of the section. Moreover, in this study, a measurement method using one lateral and two vertical potentiometers were presented to determine the lateral and vertical deflections and rotation from the coupled measurements. A detailed description of the proposed technique is discussed in Sect. 4.2.

Strain gauges were also installed to measure the variation in the longitudinal strain along the depth of the crosssection at the mid-span. A total of four strain gauges were installed on each side of the cross-section in the longitudinal direction, as shown in Fig. 6: each one on the side surfaces of the top and bottom flanges and on the onefourth and three-fourth locations of the web. When the in-

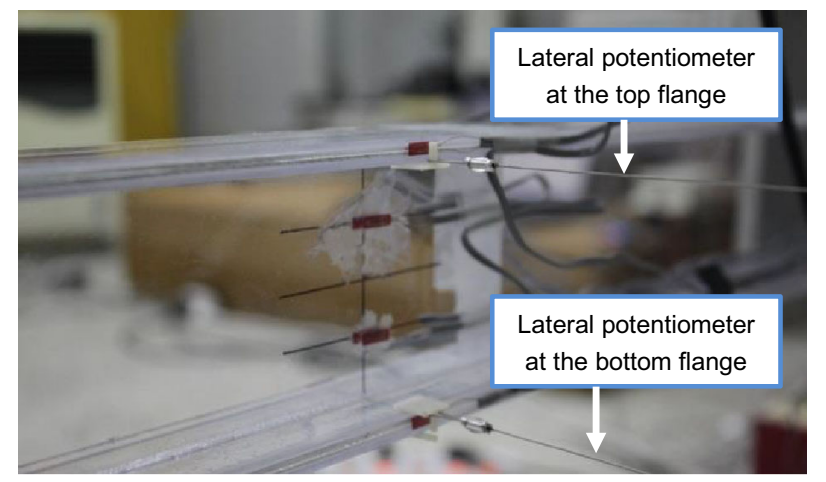

Fig. 5 Wire potentiometers for the deflection and rotation measurements.

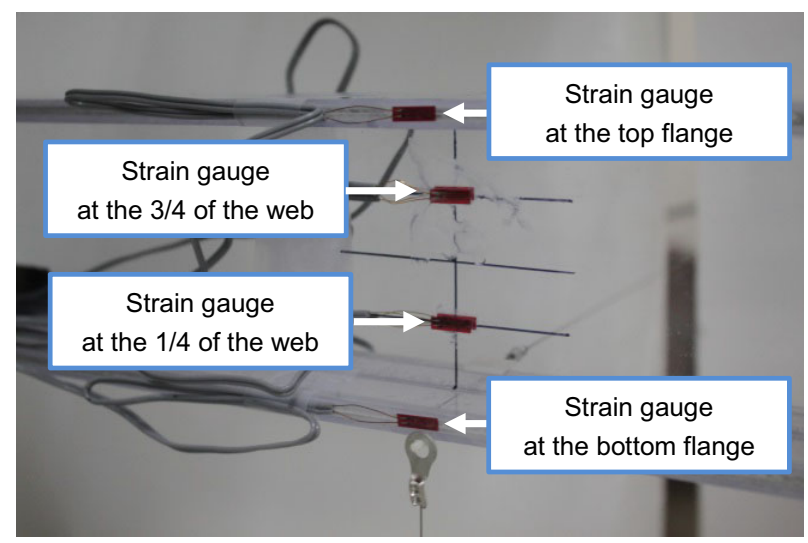

Fig. 6 Strain gauges for the strain measurements. plane flexural bending is dominant, the neutral axis of the cross-section remains horizontal. As lateral and torsional deformations occur, the neutral axis begins to rotate due to the additional tensile and compressive strains in the convex and concave parts of the beam, respectively. Therefore, the depth of the compression and tension zones, as well as the rotation of the neutral axis can be assessed using the longitudinal strain values obtained from the strain gauges.

\subsection{Method of Calculating Displacements and Rotational Angle}

The testing of lateral and torsional stability of a beam includes the lateral and vertical deflections combined with a twisting rotation of the beam. Therefore, Stoddard (1997) modified the approach proposed by Zhao et al. $(1994,1995)$ to propose a method to calculate the lateral and vertical deflections and rotation at the centroid of the cross-section (or, shear center in the doubly-symmetry section) from the coupled deflection and rotation measurements. The method proposed by Stoddard (1997) uses two potentiometers, connected horizontally to the top and bottom of a rectangular beam, and another potentiometer, connected vertically to the bottom of the beam, as depicted in Fig. 7a.

Instead of using the lateral top and bottom potentiometers, the use of two vertical potentiometers would be easier and more useful for instrumentation and data processing. Therefore, a modified measurement method was proposed to determine the twisting rotation of the cross-section. Figure $7 \mathrm{~b}$ shows the geometric configurations before and after deformation of the beam. The initial wire lengths of the one horizontal and two vertical potentiometers, $L_{o}, V_{o}$, and, $B_{o}$, changes to $L_{f}, V_{f}$, and $B_{f}$, respectively, with the deformation and rotation of the beam. Using the Pythagorean Theorem for the two triangles, shown in Fig. 7b, the vertical and lateral deformations at the bottom of the beam, $B_{x}$ and $B_{y}$, respectively, can be calculated using the following equations:

$$
\begin{aligned}
& \left(B_{o}-B_{x}\right)^{2}+B_{y}^{2}=B_{f}^{2} \\
& \left(V_{o}-B_{y}\right)^{2}+B_{x}^{2}=V_{f}^{2}
\end{aligned}
$$

Equations (1) and (2) provide two sets of solutions as follows:

$$
\left(B_{x}, B_{y}\right)=\left(\frac{B_{0} C_{1} \pm V_{0} C_{2}}{C_{3}}, \frac{V_{0} D_{1} \pm B_{0} D_{2}}{D_{3}}\right)
$$

where,

$$
\begin{aligned}
& C_{1}=B_{o}^{2}+V_{o}^{2}-B_{f}^{2}+V_{f}^{2} \\
& D_{1}=B_{o}^{2}+V_{o}^{2}+B_{f}^{2}-V_{f}^{2}
\end{aligned}
$$




$$
C_{2}=D_{2}=\sqrt{-B_{o}^{4}-B_{f}^{4}+2 B_{o}^{2} B_{f}^{2}-2 V_{o}^{2} B_{o}^{2}+2 V_{o}^{2} B_{f}^{2}-V_{o}^{4}-V_{f}^{4}+2 V_{o}^{2} V_{f}^{2}+2 V_{f}^{2} B_{o}^{2}+2 V_{f}^{2} B_{f}^{2}}
$$

$$
C_{3}=D_{3}=2\left(B_{o}^{2}+V_{o}^{2}\right)
$$

According to Stoddard (Stoddard 1997), the sets of solutions, presented in Eq. (3), have a symmetrical relationship with each other. Therefore, one set of realistic solutions should be selected to determine the lateral and vertical displacement at the bottom of the beam, at which one horizontal and vertical potentiometer was connected. Based on the determined displacements, a twisting rotation of the beam can be calculated using the Pythagorean Theorem for another triangle composed at the left side of the bottom of the beam:

$$
\left[B_{x}+b \cdot(1-\cos \phi)\right]^{2}+\left[V_{o}-B_{y}+b \cdot \sin \phi\right]^{2}=L_{f}^{2}
$$

(a)

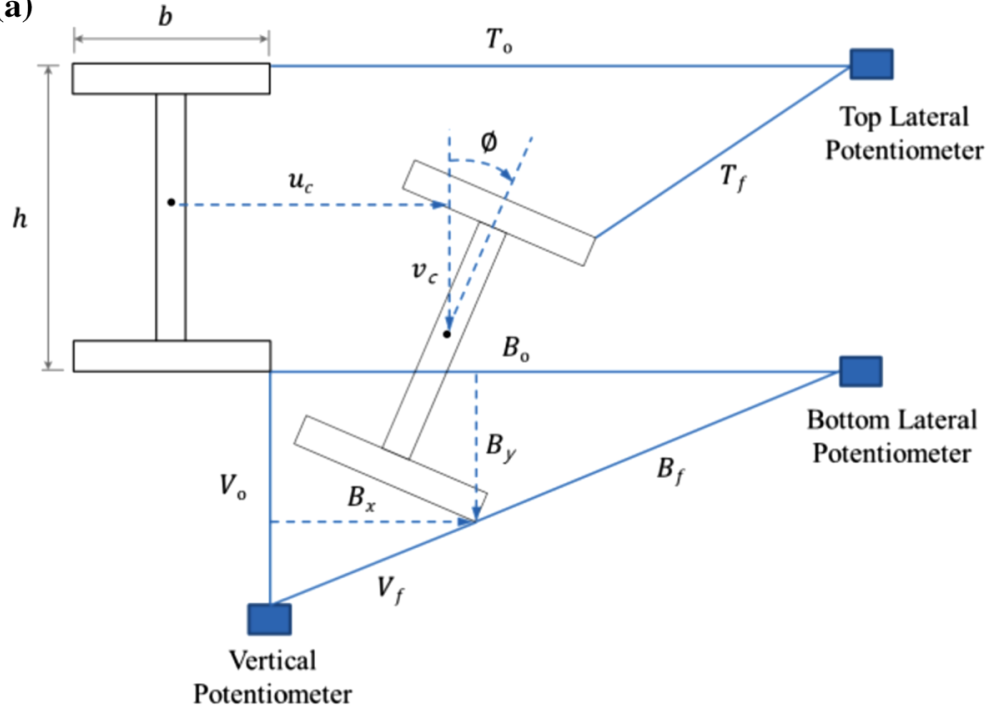

(b)

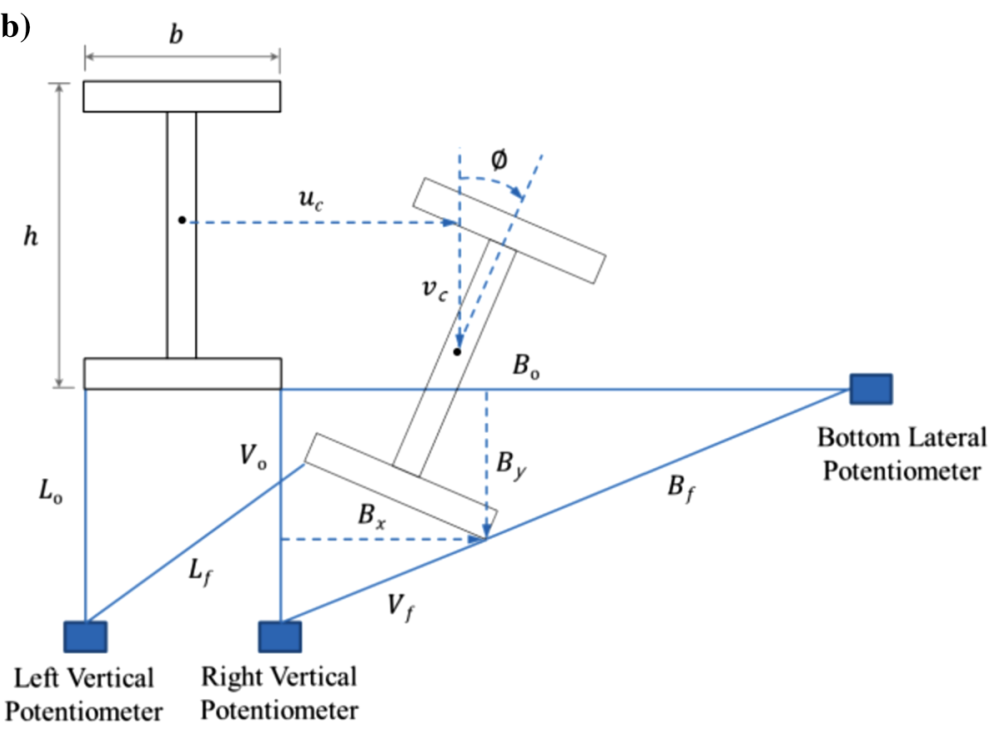

Fig. 7 Configurations of the potentiometers before and after deformation of the beam using a two lateral potentiometers and $\mathbf{b}$ two vertical potentiometers.

382 | International Journal of Concrete Structures and Materials (Vol.11, No.2, June 2017) where $\phi$ is the rotation of the beam. Equation (8) yields two solutions, as shown in Eq. (9). Similar to the solutions obtained from Eq. (3), an appropriate solution should be selected compared to the measured values.

$$
\begin{aligned}
\phi= & \left(2 \arctan \left(\frac{\sqrt{-\left(R_{1}+E_{1}\right)\left(R_{2}+E_{1}\right)}-R_{3}}{\left(R_{4}+E_{1}\right)}\right),\right. \\
& \left.-2 \arctan \frac{\sqrt{-\left(R_{1}+E_{1}\right)\left(R_{2}+E_{1}\right)}+R_{3}}{\left(R_{4}+E_{1}\right)}\right)
\end{aligned}
$$

where,

$$
E_{1}=B_{x}^{2}-2 B_{y} V_{o}+V_{o}^{2}+B_{y}^{2}-L_{f}^{2}
$$




$$
\begin{aligned}
& R_{1}=-2 b\left(L_{f}-B_{x}\right) \\
& R_{2}=2 b\left(L_{f}+B_{x}\right) \\
& R_{3}=2 b\left(B_{y}-V_{o}\right) \\
& R_{4}=2 b\left(2 b+2 B_{x}\right)
\end{aligned}
$$

Subsequently, the lateral and vertical deflections at the centroid of the cross-section, $u_{c}$ and $v_{c}$, respectively, can be calculated using the geometric relationships between the initial and final positions of the cross-section based on the obtained rotational angle of the beam, as shown in Fig. 7.

$$
\begin{aligned}
& u_{c}=B_{x}+\frac{h}{2} \sin \phi+\frac{b}{2}(1-\cos \phi) \\
& v_{c}=B_{y}+\frac{h}{2}(1-\cos \phi)-\frac{b}{2} \sin \phi
\end{aligned}
$$

The proposed procedure and equations are based on a geometrically perfect beam. When including the effects of the initial twisting angle of the cross-section, shown in Fig. 8, Eq. (8) can by expressed as

$$
\left[B_{x}+b \cdot\left(\cos \phi_{i}-\cos \phi\right)\right]^{2}+\left[V_{o}-B_{y}+b \cdot \sin \phi\right]^{2}=L_{f}^{2}
$$

Equation (17) was solved to obtain solutions similar to that of Eq. (9) with the following different terms accounting for the effects of the initial rotation:

$$
\begin{aligned}
& R_{1}=-2 b\left(L_{f}-B_{x} \cos \phi\right)+\left(\cos ^{2} \phi_{i}-1\right) b^{2} \\
& R_{2}=2 b\left(L_{f}+B_{x} \cos \phi_{i}\right)+\left(\cos ^{2} \phi_{i}-1\right) b^{2} \\
& R_{4}=b^{2}\left(1+\cos \phi_{i}\right)^{2}+2 b B_{x}\left(1+\cos \phi_{i}\right)
\end{aligned}
$$

With the twisting rotation of the beam obtained from Eq. (17), the lateral and vertical deflections at the centroid of the cross-section, including the effects of the initial rotation of the beam, can be calculated using the following equations:

$$
\begin{aligned}
& u_{c}=B_{x}+\frac{h}{2}\left(\sin \phi-\sin \phi_{i}\right)+\frac{b}{2}\left(\cos \phi_{i}-\cos \phi\right) \\
& v_{c}=B_{y}+\frac{h}{2}\left(\cos \phi_{i}-\cos \phi\right)-\frac{b}{2}\left(\sin \phi-\sin \phi_{i}\right)
\end{aligned}
$$

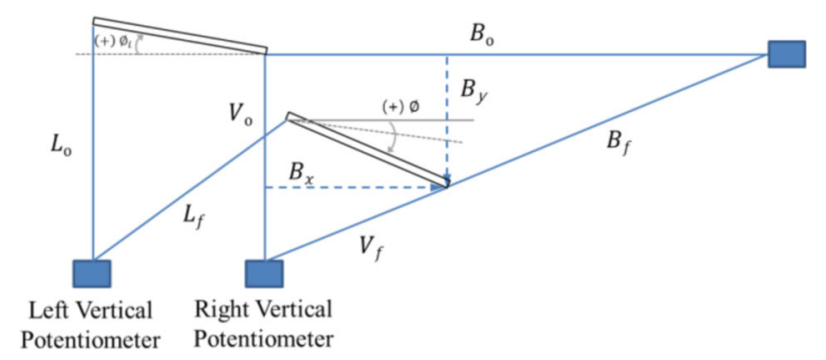

Fig. 8 Geometry configurations before and after deformation of the beam with the initial rotational angle of the cross-section.
On the other hand, when the direction of the initial and the final rotations of the cross-section are opposite, the final twisting angle can be obtained simply using Eq. (8) with no consideration of the initial rotational effect. Then, the lateral and vertical deflections at the centroid of the cross-section, which include the effects of the initial rotation of the beam, can be calculated using Eqs. (21) and (22).

\section{Experimental Results and Discussion}

\subsection{Critical Loads and Displacements of the Beam}

For two small-scaled I-beams, denoted as ST1 and ST2, a vertical point load was applied to the middle of the top flange of the beam. As determined previously, the length of the beam is $950 \mathrm{~mm}$, and the dimension of the crosssection is shown in Fig. 1b. Figures 9 and 10 show the coupled deflections measured from the wired vertical and lateral potentiometers at the mid-span. The lateral and torsional instability behavior mainly occurred in the top flange of the beams under compression. On the other hand, the bottom flange under tension was mainly dependent on the in-plane flexure. Immediately after the lateral and torsional instability occurred, the applied load was removed to ensure the elastic or inelastic buckling of the beam. As shown in Figs. 9 and 10, the lateral and torsional buckling of the small-scaled beams occurred in the linear elastic range of the material. In fact, polycarbonate, which has advantage of linear elasticity and easy manufacturing, would be good to evaluate the influence of various factors, such as geometric imperfections, support conditions, and loading system, in the lateral and torsional behavior prior to a consideration of material nonlinearity and plasticity.

From the coupled measurements, the independent vertical and lateral deflections of the beam were calculated. Figures 11 and 12 show the rotational angle of the beam

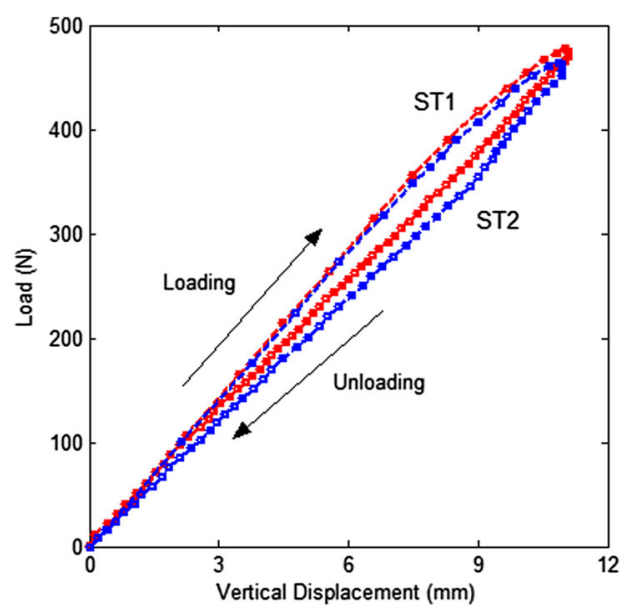

Fig. 9 Variations in the displacements at the bottom flange of the beam at the mid-span. 

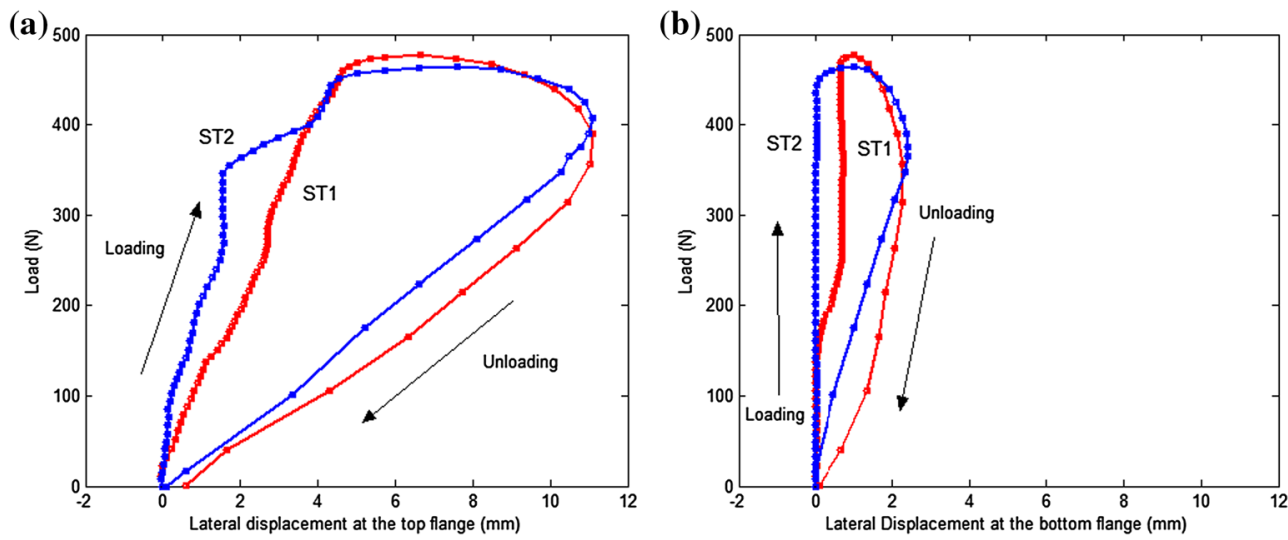

Fig. 10 Variations in the lateral displacements obtained from the horizontal potentiometers at the top and bottom flanges of the beam.

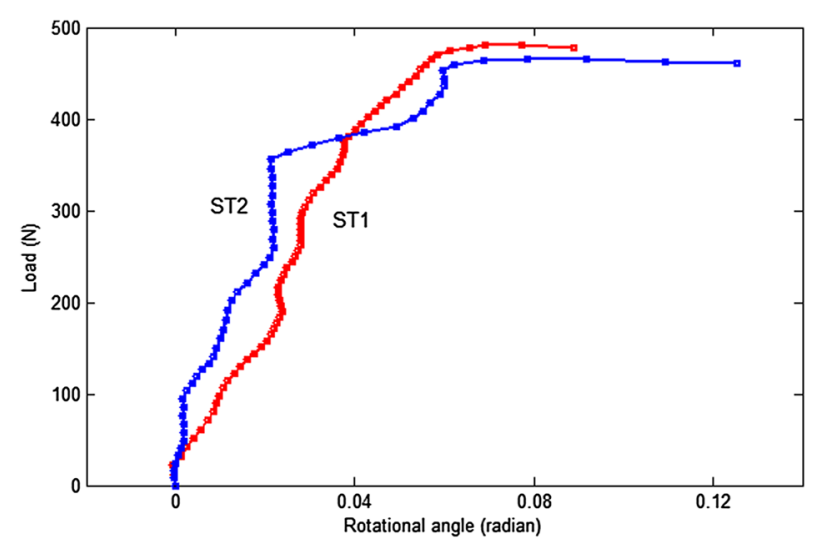

Fig. 11 Rotational angle of the beam at the mid-span.

and the lateral and vertical displacements at the centroid of the cross-section, respectively. The critical load of the beam, $P_{c r}^{\exp }$, at which the lateral and torsional instability occurred and the lateral and torsional deformations significantly increased, was determined to be approximately $473 \mathrm{~N}$ and $451 \mathrm{~N}$ for the ST 1 and ST 2 specimens, respectively. The lateral displacement and rotational angle of the beam at the centroid of the cross-section at the critical condition were approximately $3.86 \mathrm{~mm}$ and 0.0639 radians, respectively, for the ST 1 and $3.13 \mathrm{~mm}$ and 0.0616 radians, respectively for the ST 2 .

The analytical critical moment of the I-beam under pure bending conditions, including the effect of the in-plane deflection, can be calculated as (Kirby and Nethercot 1997; Chen and Lui 1987)

$$
M_{o c r}=\frac{\pi}{L} \sqrt{\frac{E I_{y} G J}{1-\left(I_{y} / I_{x}\right)}} \sqrt{1+W^{2}}
$$

where $W=\frac{\pi}{L} \sqrt{\frac{E C_{w}}{G J}}$. Because the proposed experimental method prevents the beam from warping, the critical moment calculated from Eq. (23) needs to modify the support and loading conditions using the following correction factor (Nethercot and Rockey 1971):

$$
C_{r}=\frac{1.916+1.851-0.424 W^{2}}{1+0.923-0.466 W^{2}}
$$

Therefore, the critical moment of the beam is calculated to be $100 \mathrm{kN} \mathrm{mm}$, and the critical vertical load, $P_{c r}$, is determined to be $421 \mathrm{~N}$. The modulus of elasticity of polycarbonate ranged from approximately 1100 to $1300 \mathrm{~N} / \mathrm{mm}^{2}$. In this study, an average of $1200 \mathrm{~N} / \mathrm{mm}^{2}$ was used as the modulus of elasticity for the analytical calculation.
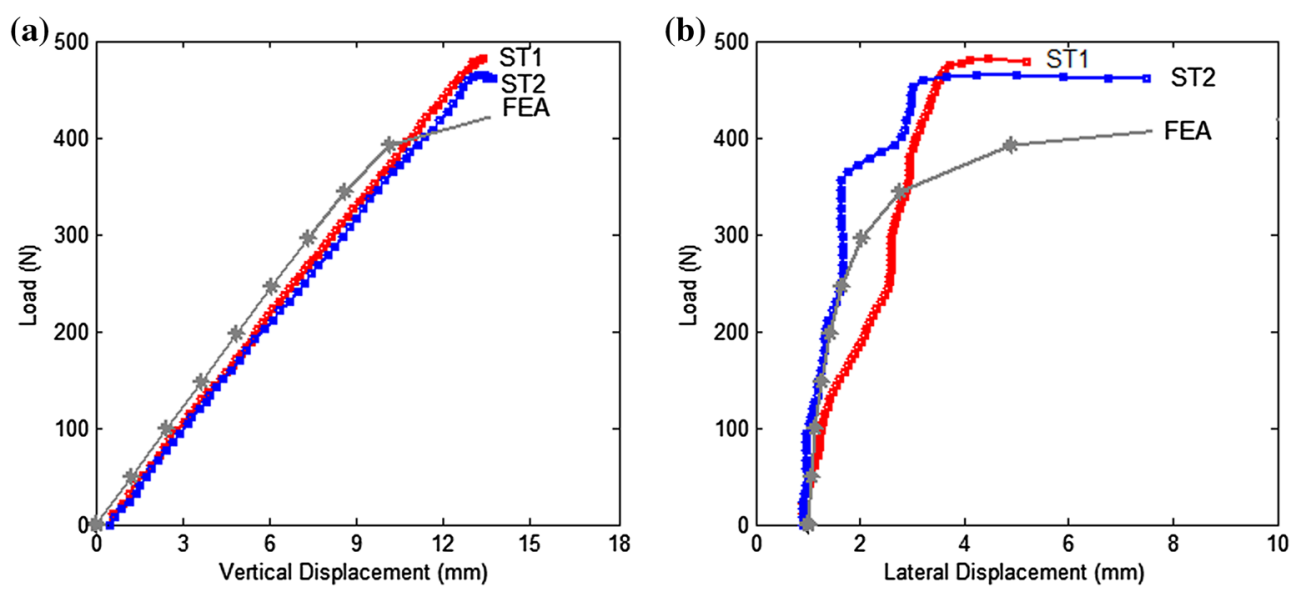

Fig. 12 Variations in the a vertical and $\mathbf{b}$ lateral displacements at the shear center of the beam at the mid-span from the experiments (ST1 and ST2) and finite element analysis (FEA). 
Compared with the critical loads obtained from the analytical equations, those obtained from the experiment was 12 and $7 \%$ larger than that from the analytical procedure for the ST 1 and ST 2 specimens, respectively. The results from the proposed experimental method were reasonably in agreement with the analytical results. Slight difference might be attributed to some frictional resistance at the contact surface of the lateral support and the side surface of the top and bottom flanges or some errors in the value of the modulus of elasticity of polycarbonate.

In addition, DIANA finite element program (DIANA 2016) was employed to compare the lateral behavior and critical load of the beam. The geometry of the beam was modeled using shell elements. To minimize the warping effect of the cross-section, beam elements with the material properties of steel were attached to the section of the beam at the both ends. The critical load obtained from the eigenvalue analysis, called as stability analysis in DIANA, was $432 \mathrm{~N}$ similar to that calculated using the analytical Eqs. (23) and (24). Furthermore, nonlinear analysis accounting for the geometric nonlinear effects (or large deformation) was also performed. The initially imperfect geometry was defined to be the first mode shape, obtained from the eigenvalue analysis, with a maximum lateral displacement of $1 \mathrm{~mm}$ at

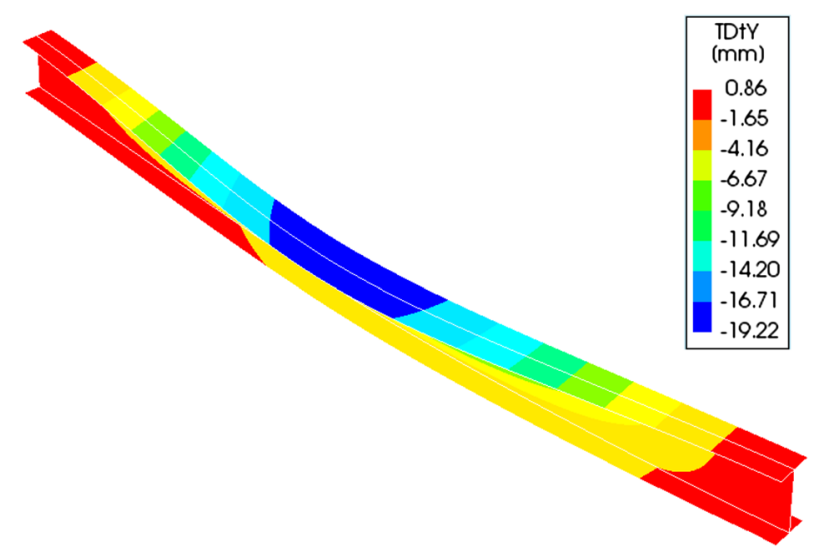

Fig. 13 Lateral displacement of the beam at the vertical load of $393 \mathrm{~N}$ obtained from the nonlinear analysis in DIANA (2016). the mid-span. Figure 13 shows the contours of the lateral displacement of the beam at the vertical load of $393 \mathrm{~N}$, at which the lateral and torsional instability occurred. The critical load obtained from the nonlinear finite element analysis (FEA) exhibited a somewhat small value, approximately 9 and $7 \%$ smaller than those of the eigenvalue analysis and analytical equations, respectively. The vertical and lateral displacements, shown in Fig. 12, were reasonably in agreement with the experimental results until the lateral and torsional instability occurred. The critical load of the experiment was approximately 17 and $13 \%$ larger than that of the nonlinear analysis for the ST1 and ST2 specimens, respectively. As mentioned previously, some difference might be caused by unexpected frictional resistance at the contact surface of the lateral support or at the location of loading.

\subsection{Strains and Neutral Axis of the Beam}

Figures 14 and 15 show variations in the longitudinal strains measured from the strain gauges for the ST1 and ST2 specimens. Since the strain gauges attached on the left side of the top flange malfunctioned during the test, the strain values at the upper part of the web (three-fourth of the height of the web), denoted as web-upper in Figs. 14 and 15, were used to compare the variations in the strains at the left and right sides (concave and convex sides, respectively) of the beam. As the load increases, the bottom flange under tension and the top flange and web-upper part under compression show an approximately linear increase in the positive and negative strains, respectively. When the load reached the lateral and torsional instability, the lateral deformation significantly increased to the right side of the beam, in which an initial lateral deformation in the beam occurred. That is, the beams were bent to the right side of the beam in the lateral direction, which formed a concave and convex in the right and left sides of the beam, respectively. Therefore, the strains in the left (concave) side of the beam turned to increase compression while those in the right (convex) side turned to increase tension, as shown in Figs. 14 and 15. When the load was completely removed, all the strains returned to an initial point with no plastic strains. Therefore, as mentioned (a)

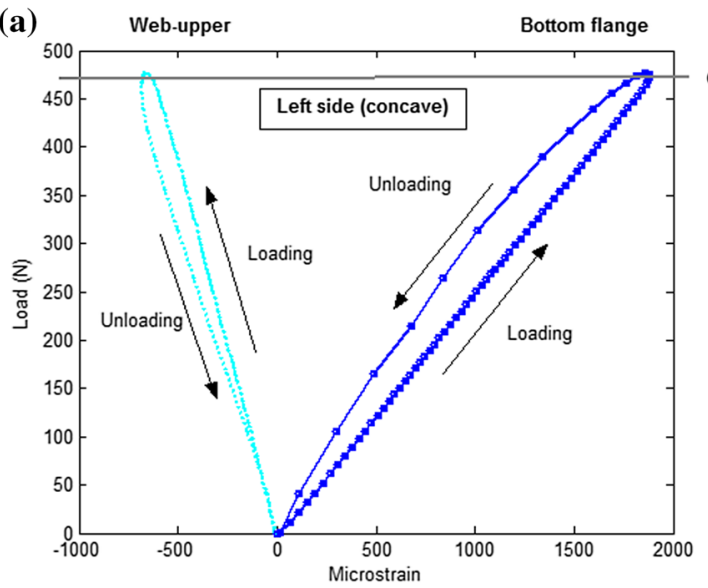

(b)

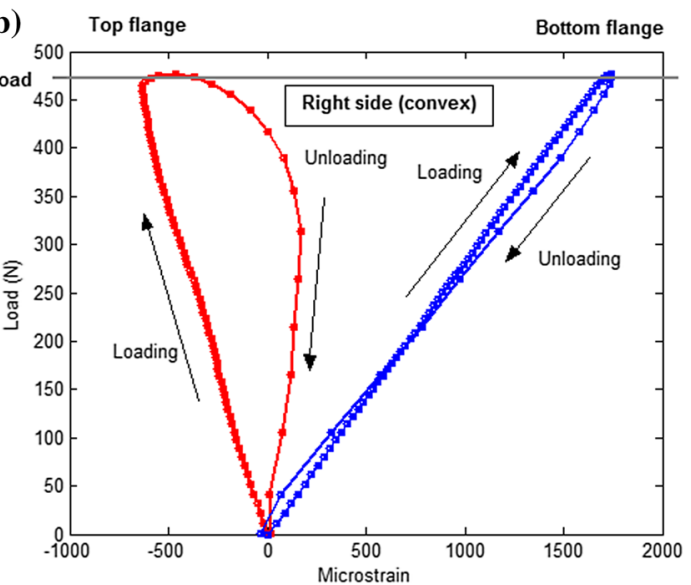

Fig. 14 Variations in the longitudinal strains measured at the a left and $\mathbf{b}$ right sides of the ST1 beam at the mid-span. 
(a)

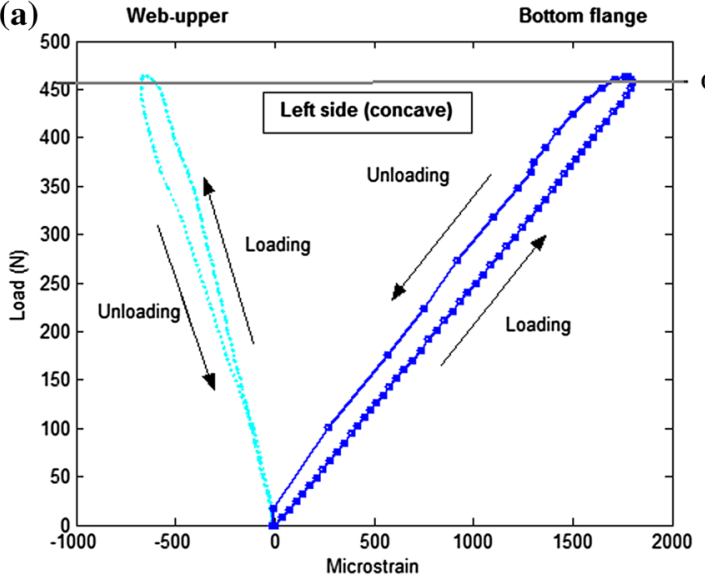

(b)

Top flange

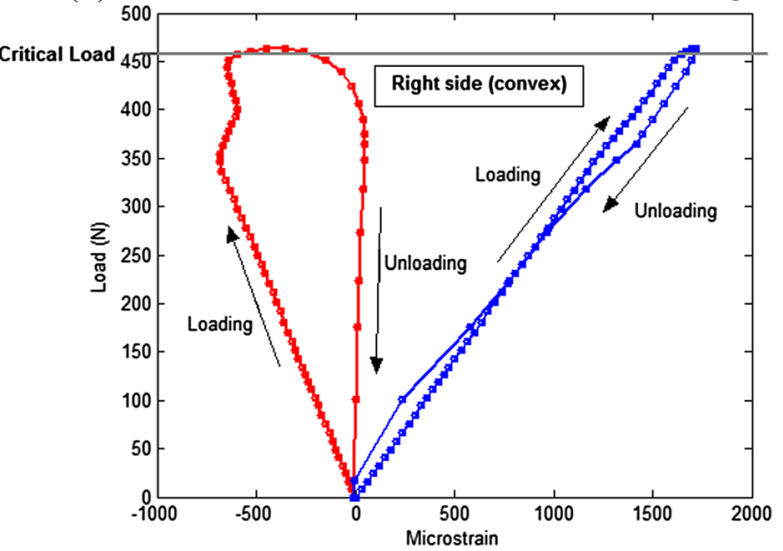

Fig. 15 Variations in the longitudinal strains measured at the a left and $\mathbf{b}$ right sides of the ST2 beam at the mid-span.

(a)

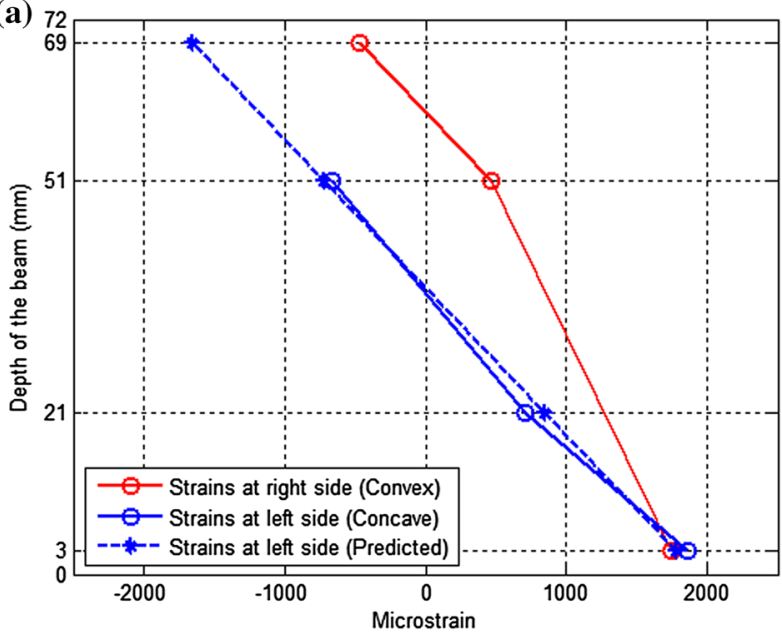

(b)

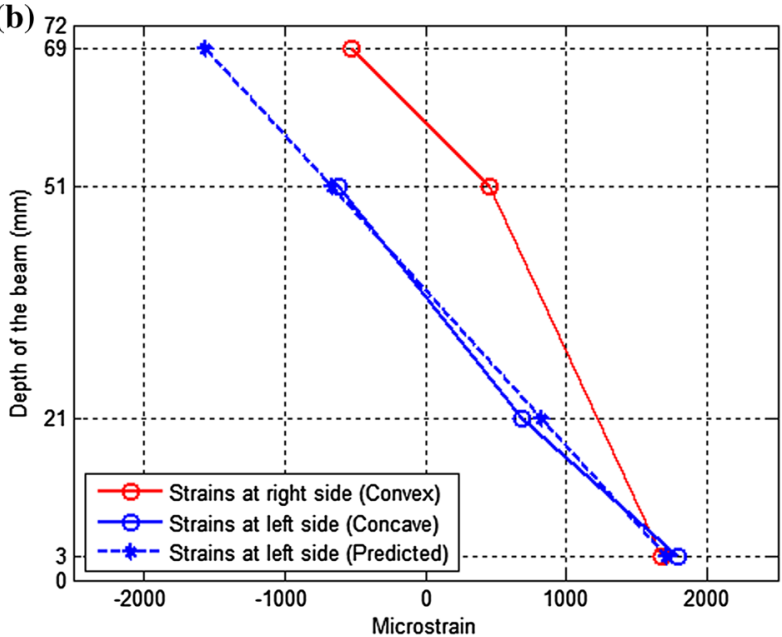

Fig. 16 Strain distribution along the depth of the a ST1 and b ST2 beams at the critical condition.

previously, the lateral and torsional instability of the beams occurred in the linear elastic range of the material.

In addition, this study examined the distribution of strains through the depth of the beam at the mid-span. Figure 16 shows the strain distribution of the ST1 and ST2 beams at the critical condition of the lateral and torsional stability. The strains at the top flange in the left (concave) side of the beam, which were not measured due to the malfunction of the strain gauges, were predicted using a liner regression analysis using the strains measured at the web and bottom flanges. As shown in Fig. 16, the measured strains are almost linearly distributed along the depth of the cross-section at both the left and right (concave and convex, respectively) sides of the cross-section. Therefore, the strains at the top flange predicted using the three strains - two strains measured at the web and one strain at the bottom flange - are expected to reasonably exhibit the actual behavior of the beam. The strains in the top and bottom flanges were under compression and tension, respectively, induced by the in-plane flexure. The tensile strains in the bottom flange were similar between the left and right sides of the section. On the other hand, the compressive strains in the right (convex) side, which decreased due to the out-of-plane flexure, were significantly smaller than those in the left (concave) side. Therefore, the bottom flange in tension was strongly dependent on the in-plane flexure, and the top flange in compression generated the lateral and torsional buckling, which decreased the compression in the right (convex) side of the section. The decrease in the compressive strains at the right (convex) side indicates that the neutral axis moves from the centroid of the cross-section to the top flange. Figure 17 illustrates the neutral axis and the corresponding compression zones of the ST1 and ST2 beams, determined from the strain profiles along the depth of the beam at the critical load. With the compression decreasing in the right (convex) side, the neutral axis moves up to the top flange, as shown in Fig. 17.

\section{Conclusions}

Lateral and torsional stability is the main issue for the safety of structures from the design and construction stages in the field of engineering. On the other hand, tests of the lateral and torsional stability are highly susceptible to the changes in the experimental conditions, such as loading and support conditions. Therefore, the lateral and torsional stability experiment, particularly using a full-size beam, requires careful consideration but is difficult to control all 

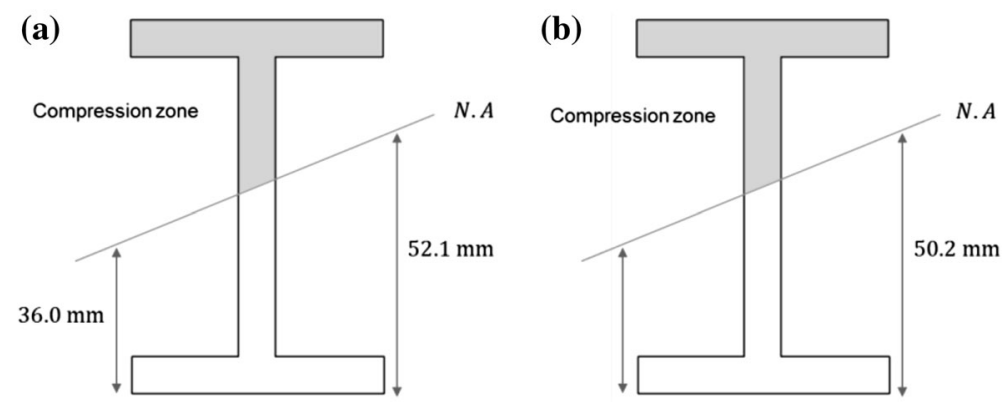

Fig. 17 Compression zones for the a ST1 and b ST2 beams at the critical condition.

these experimental conditions. That is, the slight deviation from the designated conditions could completely change the experimental results. Moreover, the lateral and vertical deformation measurements are coupled with a twisting angle of the beam. Therefore, in this study, an experimental and measurement method that can implement the experimental conditions using a small-scale model was proposed. The conclusions and recommendation proposed by this study are as follows:

- The lateral and torsional stability experiment should achieve the in-plane and out-of-plane support conditions. In this study, the simply supported condition in the longitudinal direction of the beam was implemented using a roller that restrains the vertical and lateral translations but allows in-plane flexure of the beam. The lateral support conditions, which should restrain lateral translation and twisting rotation but allow flexural rotations about the strong and weak axes of the cross-section, were provided using a ball caster and circular rod support.

- This study also provides a vertical loading system based on the roller mechanism for easy instrumentation and handling. The loading transfer system was designed to free the lateral deformation using a semicircle notch along the width of the beam and the torsional rotation with the help of the ball-and-socket joint. The proposed method has the advantage of maintaining its initially vertical and concentric position throughout the course of loading, even when the beam undergoes coupled deformation and rotation.

- The lateral and vertical deflections, combined with a twisting rotation of the beam, cannot be obtained directly from the measurement values of the potentiometers. Thus, a modified measurement method using two vertical potentiometers, which would be easier and more useful for instrumentation and data processing, was presented to determine the lateral and vertical deflections and rotation from the coupled measurements.

- For small-scaled I-beams, the critical loads obtained from the proposed experimental method were in good agreement with those calculated using the analytical equations. The strains in the top and bottom flanges, which were under compression and tension, respectively, were induced by the in-plane flexure. On the other hand, the compressive strains in the convex side of the beam decreased due to the out-of-plane flexure. Therefore, the neutral axis in the concave side moves from the centroid of the cross-section up to the top flange. The proposed experimental method, validated in the linear and elastic range of material, could be further applied to the lateral and torsional stability testing of flexural members accounting for various parameters, such as material nonlinearity, shapes and sizes of geometry, and boundary conditions.

\section{Acknowledgements}

This study was supported by Basic Science Research Program through the National Research Foundation (NRF) of Korea funded by the Ministry of Science, ICT \& Future Planning (NRF-2014R1A1A1005992).

\section{Open Access}

This article is distributed under the terms of the Creative Commons Attribution 4.0 International License (http:// creativecommons.org/licenses/by/4.0/), which permits unre stricted use, distribution, and reproduction in any medium, provided you give appropriate credit to the original author(s) and the source, provide a link to the Creative Commons license, and indicate if changes were made.

\section{References}

Brsoum, R. S., \& Gallagher, R. H. (1970). Finite element analysis of torsional and torsional flexural stability problems. International Journal for Numerical Methods in Engineering, 2(3), 335-352.

Chen, W. F., \& Lui, E. M. (1987). Structural Stability: Theory and Implementation (pp. 317-333). New York: Elsevier.

Darlmaz, K. (2011). An assumed stress hybrid finite element for buckling analysis. Mathematical and Computational Applications, 16(2), 690-701.

DIANA. (2016). TNO DIANA-finite element analysis user's manual release 10.1. Delft, The Netherlands: TNO.

Fafard, M., Beaulieu, D., \& Dhatt, G. (1987). Buckling of thinwalled members by finite elements. Computers \& Structures, 25(2), 183-190. 
Hansell, W., \& Winter, G. (1959). Lateral stability of reinforced concrete beams. ACI JouRNAL Proceedings, 56(3), 193-214.

Helwig, T. A., Wang, L., Deaver, J., \& Romero, C. (2005). Cross-frame and diaphragm behavior in bridges with skewed supports: Summary. Project Summary Report 0-1772. Austin, TX: Texas Department of Transportation.

Horne, M. R. (1954). The flexural torsional buckling of members of symmetric I-section under combined thrust and unequal terminal moments. The Quarterly Journal of Mechanics and Applied Mathematics, 7(4), 410-426.

Hou, J., \& Song, L. (2016). Progressive collapse resistance of $\mathrm{RC}$ frames under a side column removal scenario: the mechanism explained. International Journal of Concrete Structures and Materials, 10(2), 237-242.

Hurff, J. (2010). Stability of precast prestressed bridge girders considering imperfections and thermal effects. $\mathrm{PhD}$ thesis, Georgia Institute of Technology, Atlanta, GA.

Hurff, J. B., \& Kahn, L. F. (2012). Lateral-torsional buckling of structural concrete beams: experimental and analytical study. ASCE Journal of Structural Engineering, 138, $1138-1148$

Jensen, L. M. (1978). Buckling of reinforced concrete beams. Dissertation, Engineering Academy of Denmark, Copenhagen, Denmark.

Kalkan, I. (2009). Lateral torsional buckling of rectangular reinforced concrete beams. PhD thesis, Georgia Institute of Technology, Atlanta, GA.

Kalkan, I. (2014). Lateral torsional buckling of rectangular reinforced concrete beams. ACI Structural Journal, 111, $71-81$.

Kalkan, I., \& Hurff, J. B. (2012). Experimental techniques for lateral stability testing of beams. Experimental Techniques, $39,1-6$.

Kim, S. J., Kim, J. H., Yi, S. T., Noor, N. B., \& Kim, S. C. (2016). Structural performance evaluation of a precast PSC curved girder bridge constructed using multi-tasking formwork. International Journal of Concrete Structures and Materials, 10(Suppl. 3), 1-17.

Kirby, P. A., \& Nethercot, D. A. (1997). Design for structural stability. Suffolk: Granada Publishing.

König, G., \& Pauli, W. (1990). Ergebnisse von sechs Kippversuchen an schlanken Fertigteilträgern aus Stahlbeton und Spannbeton (Results of six buckling tests on slender prefabricated girders made of reinforced concrete and prestressed concrete). Beton-und Stahlbetonbau, 85(10), 253-258. (in German).

Lee, J. H. (2012a). Behavior of precast prestressed concrete bridge girders involving thermal effects and initial imperfections during construction. Engineering Structures, 42, $1-8$.

Lee, J. H. (2012b). Investigation of extreme environmental conditions and design thermal gradients during construction for prestressed concrete bridge girders. ASCE Journal of Bridge Engineering, 17(3), 547-556.

Lee, J. H., Kalkan, I., Lee, J. J., \& Cheung, J. H. (2016). Rollover instability of precast girders subjected to wind load. Mag. Conc. Res., 69(2), 68-83.
Li, J. Z., Hung, K. C., \& Cen, Z. Z. (2002). Shell element of relative degree of freedom and its application on buckling analysis of thin-walled structures. Thin-Walled Structures, $40,865-876$.

Massey, C. (1967). Lateral instability of reinforced concrete beams under uniform bending moments. ACI Journal Proceedings, 64(3), 164-172.

Nethercot, D. A., \& Rockey, K. C. (1971). A unified approach to the elastic lateral buckling of beams. The Structural Engineer, 49(7), 321-330.

Oesterle, R. G., Sheehan, M. J., Lotfi, H. R., Corley, W. G., \& Roller, J. J. (2007). Investigation of red mountain freeway bridge girder collapse. CTL Group project no. 262291 final report. Phoenix: Arizona Department of Transportation.

Petrone, F., Shan, L., \& Kunnath, S. K. (2016). Modeling of RC frame buildings for progressive collapse analysis. International Journal of Concrete Structures and Materials, 10(1), $1-13$.

Ramin, K., \& Fereidoonfar, M. (2015). Finite element modeling and nonlinear analysis for seismic assessment of off-diagonal steel braced RC frame. International Journal of Concrete Structures and Materials, 9(1), 89-118.

Rengarajan, G., Aminpour, M. A., \& Knight, N. F. (1995). Improved assumed-stress hybrid shell element with drilling degrees of freedom for linear stress, buckling and free vibration analyses. International Journal for Numerical Methods in Engineering, 28(11), 1917-1943.

Revathi, P., \& Menon, D. (2007a). Estimation of critical buckling moments in slender reinforced concrete beams. ACI Structural Journal, 103(2), 296-303.

Revathi, P., \& Menon, D. (2007b). Slenderness effects in reinforced concrete beams. ACI Structural Journal, 104(4), 412-419.

Salvadori, M. C. (1955). Lateral buckling of I-beams. ASCE Transactions, 120, 1165-1177.

Sant, J. K., \& Bletzacker, R. W. (1961). Experimental study of lateral stability of reinforced concrete beams. ACI Journal Proceedings, 58(6), 713-736.

Siev, A. (1960). The lateral buckling of slender reinforced concrete beams. Magazine of Concrete Research, 12(36), 155-164.

Srikar, G., Anand, G., \& Suriya, S. (2016). Prakash, A study on residual compression behavior of structural fiber reinforced concrete exposed to moderate temperature using digital image correlation. International Journal of Concrete Structures and Materials, 10(1), 75-85.

Stoddard, W. P. (1997). Lateral-torsional buckling behavior of polymer composite I-shaped members. PhD thesis, Georgia Institute of Technology, Atlanta, GA.

Talbot, M., \& Dhatt, G. (1987). Three discrete Kirchhoff elements for shell analysis with large geometrical non-linearities and bifurcations. Engineering with Computers, 4, 15-22.

Timoshenko, S. P. (1956). Strength of Materials: Part II (3rd ed.). Princeton: Van Nostrand Co.

Timoshenko, S. P., \& Gere, J. M. (1961). Theory of elastic stability (2nd ed.). New York: McGraw-Hill.

Yarimci, E., Yura, J. A., \& Lu, L. W. (1967). Techniques for testing structures permitted to sway. Experimental Mechanics, 7(8), 321-331. 
Yura, J. A., \& Phillips, B.A. (1992). Bracing requirements for elastic steel beams. Research Report 1239-1. Austin, TX: Center for Transportation Research, The University of Texas.

Zhao, X. L., Hancock, G. J., \& Trahair, N. S. (1994). Lateral buckling tests of cold-formed RHS beams. Research Report R699. Camperdown: School of Civil and Mining Engineering, The University of Sydney.
Zhao, X. L., Hancock, G. J., \& Trahair, N. S. (1995). Lateral buckling tests of cold-formed RHS beams. ASCE Journal of Structural Engineering, 121(11), 1565-1573.

Zureick, A. H., Kahn, L. F., \& Will, K. M. (2005). Stability of precast prestressed concrete bridge girders considering sweep and thermal effects. GDOT Project No. RP 05-15 Research Proposal. Atlanta, GA: Georgia Department of Transportation, 2005. 